\title{
Traductions transfuges, oulipotentielles
}

\section{Georgiana Lungu Badea}

Université de l'Ouest

Roumanie

Résumé : Nous exposons et examinons dans cet article les défis auxquels est confronté le traducteur de littérature potentielle. Dans le contexte de traduction et donc de reconstruction selon un principe, la contrainte mathématisable, qui réduit le nombre et les catégories de lecteurs, la tâche du traducteur est encore plus rude que dans d'autres situations traductives. La littérature des Oulipiens se dérobe aux repères traditionnels et met le traducteur à l'épreuve. Mais, pour autant, ce dernier ne sombre pas nécessairement dans l'effort de délabyrinther le texte. Le but de la traduction potentielle - partiellement défini par ses destinataires - peut offrir la clé.

Mots-clés : littérature potentielle, traduction potentielle, contrainte mathématisable, métamorphose, anamorphose, lecteur-cible

\begin{abstract}
In this paper, we present and analyse the challenges faced by the translator of potential literature. In the context of translation and thus of reconstruction according to a principle - a constraint that can be expressed mathematically, thus reducing the number and categories of readers - the translator's task is even more difficult than in other translational situations. The Oulipians' literature eludes traditional benchmarks and puts the translator to the test. However, the latter does not give in to the effort of "délabyrinther" the text.
\end{abstract}

Keywords: potential literature, potential translation, mathematically expressed constraint, metamorphosis, anamorphosis

\section{Introduction}

La traductibilité est étudiée et garantie par la thèse de la référentialité. Son absence mènerait logiquement à l'intraduisibilité d'un texte. L'illusion référentielle que les écrivains modernistes ont pratiquée « de manière visiblement ironique » (Saint-Gelais 2011, 14) ajoute encore un défi aux traducteurs de la littérature potentielle. Dans 
ce qui suit, nous nous contentons d'envisager l'une des relations extérieures qu'un texte - de Perec, en l'occurrence - peut avoir avec un autre ou d'autres texte(s), à savoir, la relation textuelle et traductionnelle, et non pas la relation fictionnelle, qui le mettrait en parallèle avec la vie réelle. "Où le roman s'achève et chacun s'en va vaquer aux occupations qui lui sont propres" (Roubaud 1990), le traducteur métamorphosiste, pour ainsi dire, continue de redire et récrire l'histoire dans une autre langue.

\section{Métamorphose ou anamorphose?}

La métamorphose traductionnelle indique la relation de transformation du texte à traduire ; par contre, l'anamorphose indique sa déformation. Tel est le contexte d'écriture perecquienne, et il nous semble essentiel pour les études de traduction de la littérature potentielle d'observer à quel point la traduction de cette littérature se situe surtout dans le champ de l'anamorphose plutôt que dans celui de la métamorphose. ${ }^{1}$ Sans se questionner inlassablement sur le "sens de[s] constructions et de[s] quêtes » (Burgelin 1988, 13), ces défis sont difficilement surmontables pour tout traducteur.

À la «virtuosité oulipienne » (Burgelin 1988, 13) de Perec, les traducteurs s'efforcent de répondre avec un talent traductionnel à la mesure des attentes. Pour y correspondre, les traducteurs se doivent de mettre en œuvre : montage et démontage, torsions et distorsions du langage, tours et détours, fonte, défonte et refonte des idées (ou, « derridiennement » parlant : construction, dé- et re-).

Situation de création vs. Situation de re-création (= traduction)

Avant d'essayer une réponse à la question « Peut-on envisager des similitudes entre l'écriture perecquienne et sa traduction? », on devrait prendre en considération la (para)logique de la traduction d'une écriture expérimentale et transfuge telle que celle de Perec et telle qu'elle est définie par Richard de Saint-Gelais (2011).

Le grand mérite de Georges Perec, c'est de n'écrire jamais deux livres semblables. Nous y reprenons un poncif de la critique, selon laquelle - la réalité le confirme - chacun de ses livres représente une nouvelle «formule », une " manière » neuve, un « système » différent des précédents et des livres à venir (in Burgelin 1988, 16). L'écriture traductologique peut reprendre ce poncif romanesque et développer des scénarios sur les affres du traducteur. Quelque vaillant qu'il fût, lequel des traducteurs voulait traduire plus d'un livre? Satisfaire, d'abord, le «foisonnement de recherches rhétorico-oulipiennes » (Burgelin 1988, 23) et plonger, ensuite, dans le système de la languecible pour les refaire, les reproduire, les rendre. Et, non pas en dernier

${ }^{1}$ Le sujet de la traduction de la littérature expérimentale a fait l'objet d'une série d'études que nous avons consacrées au livre Le Mot sablier de Dumitru Tsepeneag (Lungu Badea 2007, 2009). 
lieu, résister à la tentation de se rendre, car le plaisir de Perec de multiplier les jeux fait le déplaisir du traducteur de les re-multiplier.

Perec l'auteur disparait-il ou se retrouve-t-il dans la traduction de son livre La Disparition - un lipogramme où la parole « s'enlabyrinthe et se délabyrinthe avec tant de virtuosité » (Burgelin 1988, 35) ? Les propos de Perec à l'égard de son écriture : " cette écriture carapace derrière laquelle [il] masquai[t] [s]on désir d'écriture » annonçaient-ils ce qui devait se produire s'il disparaissait ? La tâche du traducteur serait bien accomplie. Des rêves...

Des rêves, "rêvés pour être textes ", tout comme les premières peintures rupestres représentant les hallucinations lors des transes induites par des plantes psychotropes. Les rêves racontés ou écrits, décrits, représentent déjà une traduction, du mental au verbal, à l'écrit.

\section{oulipème}

Contrainte mathématisable, unité de traduction,

Le texte oulipien à traduire subit donc une double métamorphose/anamorphose : celle que pratique l'auteur et celle que le traducteur est obligé de mettre en œuvre. On y observe des pratiques hypertextuelles (Genette 1982, 49), à fonction plus ou moins ludique (parodie et/ou transposition, pastiche ou réécriture, transformation et imitation), mais certainement recherchée. Quelle stratégie de traduction pour l'écriture oulipienne ? Mais avant tout, précisions : à qui s'adresse l'écriture potentielle; quel est son public, source ou cible. Apprentis écrivains, apprentis traducteurs, verbicrucistes, lecteurs désireux de décrypter les œuvres déconcertants des Oulipiens, caslimite et cas-modèle, et, donc, de les contresigner.

Si l'on traduit les réseaux sémantiques et syntaxiques par les équivalents lexicaux et syntagmatiques qui conviennent, on n'a qu'une pseudo-traduction, si l'on traduit le contenu sémantique sans tenir compte de sa forme (signifiant), on ne restitue que des messages, à un effet perlocutoire manqué; si l'on accepte de mettre en valeur le potentiel de la langue-cible, on doit choisir l'anamorphose (à voir les quinze micro-traductions intralinguales que Perec propose au texte de Paul Verlaine, "Gaspard Hauser chanter » qui témoigne de l'irrespect parfait du TS) au détriment de la métamorphose.

Ensuite, il faut rappeler que les oulipèmes (tels les culturèmes) doivent être traités et traduits comme des unités de traduction insécables, non divisibles sémantiquement bien que divisibles syntaxiquement.

De l'écriture traductologique à la pratique de traduction, le chemin est bien long. Pour illustrer la complexité du phénomène (de réception et de traduction), prenons donc le premier paragraphe du $5^{\mathrm{e}}$ chapitre du roman lipogrammatique, La Disparition (de la voyelle « $\mathrm{e} »)$ : 
TS : Anton Voyl n’arrivait pas à dormir. Il alluma. Son Jaz marquait minuit vingt. Il poussa un profond soupir, s'assit dans son lit, s'appuyant sur son polochon. Il prit un roman, il l'ouvrit, il lut ; mais il n'y saisissait qu'un imbroglio confus, il butait à tout instant sur un mot dont il ignorait la signification. (La Disparition, 1969)

L'examen des traductions roumaine et espagnole mène à identification de la stratégie de traduction choisie.

TC (es) : Tonio Vocel no concilió el sueño. Encendió el fluorescente. Miró el reloj: cinco y quince. Suspiró hondo, se sentó en el lecho, se reclinó sobre el cojín. Cogió un libro, lo hojeó y lo leyó; pero sólo pudo ver un lío enorme; los términos confusos le impidieron seguir el hilo. (El Secuestro, 2010, 15)

TC (roum)² : Obosit, dar fără somn, Anton Voyl a aprins lampa: oo.20. A suspinat adânc, s-a mai foit, potrivindu-şi căpătâiul gros sub cap. A luat în mână un roman. L-a răsfoit, dând a-l citi. Găsi că-i un imbroglio confuz, şi cam atât. La tot pasu-l agasa o vorbă, un vocabul, a cărui noimă n-o ştia. (Dispariția, 2010, 17).

Les possibilités de rendre en LC le lipogramme sont :

- la traduction sémantique (sémantisée)

- ou la traduction de la lettre (le mot, par correspondance ou équivalence lexicale).

Les deux versions russes du livre de Georges Perec sont à prendre en considération, illustrant chacune les stratégies de traduction décrites antérieurement. Le traducteur A. Astachonok-Jghirovski (Isceznovenie, 2001), met en œuvre une stratégie pragma-sémantique, l'acceptabilité et le lecteur-cible devenant les instances vers lesquelles il décide de faire preuve de fidélité. V. M. Kislov (Ischezanie, 2005), répond au défi que l'écrivain lance et traduit le roman-lipogramme par un équivalent russe d'où disparaît la lettre « $\mathrm{O}$ ».

Si l'emprunt ne sert à rien, le calque rend au moins le principe de «fabrication » des mots, phrases, textes; alors que la traduction littérale - comme stratégie - consisterait dans des équivalences syntagmatiques caractéristiques à la LC, la transposition ne servira qu'à obtenir des équivalences sémantiques, sans se soucier de la forme qui est ré-catégorisée ; la modulation modifiera le point de vue de l'auteur, donc il n'y aura de fidélité ni à l'intention de l'auteur ni à l'intention du

\footnotetext{
${ }^{2}$ Il est méritoire de mentionner aussi la version (2013) de Ramona Diaconu, étudiante en master de traduction. La traductrice respecte le caractère lipogrammatique du TS. « Anton Voyl nu izbuti să adoarmă. Porni lumina. Jaz-ul său indica ora oo:20. Oftă adânc, stătu în pat, pilota fiindu-i proptă. Luă un roman şi porni a citi; însă, şi păru totul confuz, întâlnind continuu un cuvânt al cărui tâlc nu îl ş̧tia. Lăsă romanul în pat. Ajuns la lavoar, udă o mănuşă, apoi îşi jilăvi fața şi gâtul. Pulsu-i zbuciuma. Căldura îl năpădi. Crăpă fanta odăii, scrută abisul nopții. îl întâmpină un văzduh plăcut. Un zgomot indistinct urca din foburg. » C'est toujours la lettre « e » qui disparait.
} 
TS ; l'adaptation dans l'écriture à contrainte, serait la pire des voix à suivre, le pire des choix à faire : une naturalisation maladroite.

La traduction comparée des versions espagnole et roumaine permet de remarquer qu'elles sont délibérément formelles et nécessairement sémantiques; respectueuses de l'intentionnalité écrivante et du principe de construction du texte-source (la voyelle « a » disparait en espagnol, la voyelle « e », en roumain, ce qui, hasard objectif ou non, correspond au choix d'origine). Les différences d'intentionnalité psychologique (de l'auteur) et sémantique (du TS) et sémantique des textes-cibles sont incontournables.

Nous avons procédé à la retro-traduction et à la retraduction de l'incipit. Cet exercice a montré que, outre la quantification des entropies sémantique, syntaxique, stylistique, informationnelle, etc., traduire la dérivation, cette " chaîne de traductions » successives dans différentes LC avant de retraduire dans la langue-source, tout comme l'aller-retour, c'est-à-dire la rétrotraduction (LS-LC-LS), n'aide en rien ni le traducteur, ni l'auteur, ni le public-cible. Néanmoins, elle offre au comparatiste la possibilité d'estimer le «décalage » sémantique, formel, intentionnel.

Étant donné qu' « il n'y a de contrainte oulipienne, au sens strict, que mathématisable » (Roubaud 1991, 82), soulignons encore une fois que toutes ces réfutations ne peuvent être prises en considération que par rapport à la poétique et à l'esthétique de réception (directe ou par la traduction).

\section{Motivations pour (ne pas) traduire la littérature expérimentale}

Les imitations complexes auxquelles se livre Perec défigurent les modèles proposés pour être reconnus (les quinze micro-traductions, des traductions internes, du poème de Verlaine, par exemple) à travers un jeu littéraire qui s'apparente à l'anamorphose littéraire, et proposent un reflet déformé du texte d'origine.

On y revient sur la question essentielle de la subjectivité de l'expression, mais notamment sur celle de la réception : et si le texte perecquien était comme une anamorphose et il changeait de sens selon le point que l'on choisit pour le regarder ? (J.-P. Néraudau 1989, 161163 in Jouteur 2011, 344). Les limites de traduction de l'exercice textuel postmoderne sont données par :

- l'absence de finalité. Ce n'est que l'écriture qui compte. Même si ce point de vue est contesté par les partisans de la théorie de l'action, selon lesquels il n'a pas d'action sans finalité ;

- l'instauration d'une empathie démotivante ;

- la polyphonie et la proche parenté de la traduction de ce texte et de la traduction de poésie par la prééminence de la forme au détriment du contenu. Le sens n'est pas pour autant étouffé parce qu'il naît au fur et à mesure que la pensée se matérialise. 
Les ratures ne sont que des façonnages d'idées, des bouts de phrases couchés sur le papier.

- l'intertextualité et l'interlingual introduisent un nouveau mode de lecture, qui diffère de la lecture linéaire: la lecture alternative. Serait-il insensé de parler de traduction alternative?

\section{Essais de tirer une conclusion}

À cette traduction intrapersonnelle, aux différentes formes de traduction invoquées par les Oulipiens - traductions homophoniques, syntaxiques et homosémantiques (cf. Atlas de littérature potentielle, 1988 : 143-158) - le traducteur, croyons nous, doit répondre fermement par l'identification et la maîtrise de la contrainte (soit-elle visible ou invisible) et de la "révélation » (comme se plaisent à l'appeler Italo Calvino et d'autres Oulipiens). Arrivés ici, nous ne pouvons pas ignorer une question légitime : le traducteur doit-il faire preuve d'inspiration dans la traduction de l'écriture à contrainte ou il suffit qu'il soit un bon technicien de la langue, un fabricant de textes selon des principes mathématiques?

Révision d'une conclusion ouverte. Un texte a un commencement et une fin. Un hypertexte n'a ni commencement ni fin. Il est panoramique. Lire un mot le met en mouvement, attirant dans son jeu le créateur et le lecteur ; il s'offre à eux et, épuisant l'énergie suggestive, créatrice, il s'épuise. Le traducteur oulipotentiel accepte le défi, joue le jeu, le déjoue et rejoue pour donner l'occasion au lecteurcible de s'enlabyrinther à son gré.

\section{Références bibliographiques}

Burgelin, C. Georges Perec. Paris : Éditions du Seuil, coll. «Les contemporains », 1988.

Collombat, Isabelle. "L'Oulipo du traducteur». In: Semen [En ligne], 19/2005, mis en ligne le 16 mai 2007, consulté le 14 octobre 2011. URL : http://semen.revues.org/2143.

Dupriez, Bernard. Les procédés littéraires, 2004.

Eliade, Mircea. «Lumière et transcendance dans l'œuvre d'Eugène Ionesco ». In: Ionesco. Situation et perspectives, sous la direction de Marie-France Ionesco et Paul Vernois, Colloque de Cerisy-la Salle, août 1978. Paris : Éditions Belfond, 1980 : 117-127.

Jouteur, Isabelle. Dying, Death and Bereavement in a British Hindu Community. Leuven: Peeters, 2001.

Genette, Gérard. Palimpsestes : la littérature au second degré. Paris : Éditions du Seuil, 1982.

Lungu Badea, Georgiana. D. Tsepeneag et le régime des mots. Écrire et traduire "en dehors de chez soi ». Timişoara : Editura Universității de Vest, 2009. 
Lungu-Badea, Georgiana. «An (In)Visible Bridge: From Mental To Interlingual Translation. Reflections On Translating The Experimental Writings Of Dumitru Tsepeneag ». In: Sanda Badescu (ed.). From One Shore to Another. Reflections on the Symbolism of the Bridges. Cambridge: Cambridge Scholars Publishing, 2007: 72-86.

Lungu-Badea, Georgiana. " Un minimalist înrăit: Dumitru Țepeneag ». Propos recueillis par Georgiana Lungu Badea, dans Orizont $\mathrm{n}^{\circ} 10$ (1465), série nouvelle, XVI; 20 oct. /2004, p. 4-5, réédition «Un minimaliste acharné : Dumitru Tsepeneag ». Traduit du roumain par Andreea Gheorghiu. In : Dialogues francophones, Timişoara, Editura Universității de Vest, $\mathrm{n}^{\circ}{ }_{12} / 2006$, p. 199-208.

Mihailescu, Dan C. «L'Architecture de l'enfance ». In : Lectures de Ionesco, Textes réunis par Norbert Dodille, Marie-France Ionesco et Gabriel Liiceanu, préface de Marie-France Ionesco, Paris, L'Harmattan et l'Institut Français de Bucarest, 2006 : 81-85.

OULIPO, Atlas de littérature potentielle. Paris : Gallimard, 1988.

Perec, Georges. "Micro-traductions, 15 variations discrètes sur un poème connu ». In : Change $\mathrm{n}^{\circ}$ 14/1973, Transformer traduire, pp. 113-117.

Roubaud, Jacques. L'Exil d'Hortense. Paris : Seghers, 1990.

Saint-Gelais, Richard. Les Fictions transfuges. La fictionnalité et ses enjeux. Paris : Editions du Seuil, 2011.

\section{Corpus}

Perec, Georges. La Disparition. Paris : Denoël, 1969.

Perec, Georges. El secuestro. Traduit du français par Marisol Arbués, Mercè Burrel, Marc Parayre, Hermes Salceda. Regina Vega, $1^{\mathrm{e}}$ édition 1997, $2^{\mathrm{e}}$ édition 2010.

Perec, Georges. Dispariția. Traducere de Şerban Foarță, Editura Art, Colecția „Biblioteca Ideală”, 2010.

Perec, Georges. Isceznovenie. Traduction en russe d'A. Astachonok-Jghirovski. Kiev : Ed. Nika-Tsenter, 2001.

Perec, Georges. Ischezanie. Traduction en russe de Kislov, V. M., St. Petersbourg : Ed. Ivan Limbach, 2005. 